\title{
Modeling the rare diseases process in dish
}

\author{
Wei Tang* \\ National Center for Global Health and Medicine, Tokyo, Japan.
}

\begin{abstract}
Summary For rare diseases, how to mimic the pathological progression is of importance for better understanding the molecular mechanism and identifying potential targets. Induced pluripotent stem cells (iPSCs) technology provides an ideal model to in vitro obtain the diseases-associated cells. In this issue of Intractable \& Rare Diseases Research, two birth detect diseases iPS models on phenylketonuria and trisomy 18 were established, and have demonstrated great potential in the research on these rare diseases.
\end{abstract}

Keywords: Rare diseases, cell model, induced pluripotent stem cells, phenylketonuria, trisomy 18

The opportunity of obtaining pathological samples from a rare disease patient is extremely rare. Therefore, most of the current researches focus on the gene knockout mice models, however, what extent of these models can reflect the aspects of rare diseases is always difficult to be evaluated accurately.

During the past decade, the development of induced pluripotent stem cells (iPSCs) technology provides an overall new strategy to reproduce the pathological progress of diseases in a dish (1). Human iPSCs can derived from many obtained somatic cells from patients such as surgically resected skin fibroblasts, blood mononuclear cells, urine cells or body liquid cells, et al. The iPSCs from rare disease patients have the capacity for self-renewal and pluripotency, theoretically are well suited to modeling the disease process of donors. Using the iPSCs-based models, a series of new molecular mechanisms of rare diseases has been identified (2). These in dish rare disease models are superior to other strategies in the following aspects: first, the most diseaseassociated cell types can be obtained step by step from iPSCs; secondly, the cell models carry all the genetic and even part of epigenetic information of the patients; thirdly, donor sources of iPSCs are safely, affordably, and always frequently obtained.

In this current issue, two original articles described their work on the establishment of rare diseases models. Xing et al. (3) induced a typical human trisomy 18

\footnotetext{
*Address correspondence to:

Dr. Wei Tang, National Center for Global Health and Medicine, Japan. 1-21-1,Toyama Shinjuku-ku, Tokyo 162-8655 Japan.
}

E-mail: politang-tky@umin.ac.jp induced pluripotent stem cell line from amniotic fluid cells. Different previous unsuccessful effort, this model retained stable trisomy 18 trait after 1ong generation of passages. In another paper, Qi et al. (4) generated a urine-derived iPSCs from a patient with Phenylketonuria (PKU). PKU-iPSCs have been established previously, this is the first time PKU-iPSCs were induced from the urine cells of PKU patients.

The use of patient-specific iPSCs for modeling rare diseases has demonstrated its potential to provide a better understanding of the exact pathological mechanisms of rare diseases, and is becoming more routine and more scalable. Intractable \& Rare Diseases Research will continue to focus on more efforts to introduce most updated knowledge and original research in this field in the future.

\section{References}

1. Shi Y, Inoue H, Wu JC, Yamanaka S. Induced pluripotent stem cell technology: a decade of progress. Nat Rev Drug Discov. 2017; 16:115-130.

2. Anderson RH, Francis KR. Modeling rare diseases with induced pluripotent stem cell technology. Mol Cell Probes. 2018; pii: S0890-8508(18)30001-X.

3. Xing KX, Cui YZ, Luan J, Zhou XY, Shi L, Han JX. Establishment of a human trisomy 18 induced pluripotent stem cell line from amniotic fluid cells. Intractable Rare Dis Res. 2018; 7:94-99.

4. Qi ZJ, Cui YZ, Shi L, Luan J, Zhou XY, Han JX. Generation of urine-derived induced pluripotent stem cells from a patient with phenylketonuria. Intractable Rare Dis Res. 2018; 7:87-93.

(Received May 25, 2018; Accepted May 28, 2018) 